\title{
THE EXCHANGE FORMULA FOR DISTRIBUTIONS AND SPANS OF TRANSLATES
}

\author{
R. E. EDWARDS
}

1. The aims of this paper are modest. Let $R^{m}$ denote real Euclidean space of $m$ dimensions with points $x=\left(x_{1}, \cdots, x_{m}\right)$; we look upon $R^{m}$ as an additive locally compact abelian group whose dual is isomorphic with $R^{m}$. We reserve the notation $y=\left(y_{1}, \cdots, y_{m}\right)$ for points of $R^{m}$ when regarded as elements of the dual. Concerning distributions on $R^{m}$, we adopt the notation of Schwartz [1]. In the theory of the generalised Fourier transform there are two basic "exchange formulae" $[1$, vol. 2, p. 124] in which are specified mutual relationships between two temperate distributions $u$ and $v$ with transforms $U$ and $V$ in order that either or both of the relations

$$
\mathcal{F}(u v)=U * V
$$

and

$$
\mathcal{F}(u * v)=U \cdot V
$$

be valid. In this paper we first observe certain other sets of conditions under which such formulae hold, and then use these formulae to study spans of translates in $L^{p}\left(R^{m}\right)$.

It seems a priori very unlikely that the best possible results concerning the latter problem will appear from such general arguments. Nevertheless, it is felt that the results obtainable in this fairly general fashion are sufficiently far from being trivial to justify a record of the procedure.

The method appeared first in an attempt by the author to apply the method of distributions to the $L^{1}$-problem in one dimension and was further clarified through personal correspondence with $M$. Schwartz.

The notation being always that of [1], let $a$ and $b$ be distributions on $R^{m}$ with transforms $A$ and $B$ respectively. In all the applications we contemplate, the convolution $a * b$ exists as a bounded function, and we are interested in the validity of the formula

$$
\mathcal{F}(a * b)=A \cdot B \text {. }
$$

Let $R_{n}(y)(n=1,2, \cdots)$ be any regularising sequence, let $A_{n}=A$ * $R_{n}$, and let $r_{n}(x)=\overline{\mathcal{F}}\left(R_{n}\right)$. By [1, vol. 2, p. 95], $A_{n} \in\left(\mathcal{\Theta}_{M}\right)$. Hence by equation (VII, $8 ; 4)$ of $[1$, vol. 2, p. 124] (with $\mathcal{F}$ replaced by $\overline{\mathcal{F}}$ ),

Received by the editors September 19, 1952. 


$$
\overline{\mathcal{F}}\left(A_{n} \cdot B\right)=\overline{\mathcal{F}}\left(A_{n}\right) * \overline{\mathcal{F}}(B) .
$$

By equation (VII, 8; 5), loc. cit.,

$$
\overline{\mathcal{f}}\left(A_{n}\right)=\overline{\mathcal{F}}(A) \cdot \overline{\mathcal{f}}\left(R_{n}\right)=a r_{n} .
$$

Since also $\mathcal{F}(B)=b$, we have $\overline{\mathcal{F}}\left(A_{n} B\right)=\left(r_{n} a\right) * b$, or equivalently

$$
A_{n} B=\mathcal{F}\left\{\left(R_{n} a\right) * b\right\} .
$$

It follows that (1.3) will be true provided that:

$$
\left(A * R_{n}\right) B \rightarrow A \cdot B \text { and }\left(r_{n} a\right) * b \rightarrow b,
$$

the one case in the sense of $\left(\Phi^{\prime}\right)$, and the other in the sense of $\left(S^{\prime}\right)$.

This type of argument was suggested independently by Schwartz in a personal letter to the author.

2. Special cases of (1.3). As a first special case, assume that $a=f$, a function in $L^{1}\left(R^{m}\right)$, and that $b=\phi$, a function in $L^{\infty}\left(R^{m}\right)$. Here $f * \phi$ exists as a function in $L^{\infty}\left(R^{m}\right)$. Further, $\left(r_{n} f\right) * \phi \rightarrow f * \phi$ in $\left(S^{\prime}\right)$ : in fact, $r_{n} \rightarrow 1$ boundedly and uniformly on each bounded set, hence $r_{n} f \rightarrow f$ in $L^{1}$, and so $\left(r_{n} f\right) * \phi \rightarrow f * \phi$ in $L^{\infty}$, a fortiori in $\left(S^{\prime}\right)$. Further, it is easy to estimate an integer $k_{m, 1}$ such that, for any $\phi \in L^{\infty}$, $\Phi=\mathfrak{F}(\phi)$ is a distribution of order at most $k_{m, 1}$. Concerning the numerical estimate of these and other integers, see the end of this section. It results that $\mathcal{f}(f * \phi)=F \cdot \Phi$ provided $F \in\left(\mathcal{E}^{k_{m, 1}}\right)$, since this last condition ensures that $\left(F * R_{n}\right) \cdot \Phi \rightarrow F \cdot \Phi$ in $\left(\Phi^{\prime}\right)$ in view of the fact that $F * R_{n} \rightarrow F$ and $D^{q}\left(F * R_{n}\right) \rightarrow D^{q} F$, in each case uniformly on each bounded set, for each symbol of derivation $D^{q}$ of rank $\leqq k_{m, 1 .}{ }^{1}$

A similar argument deals with the case in which $f \in L^{p}\left(R^{m}\right)$ and $\phi \in L^{p^{\prime}}\left(R^{m}\right)$, where $1 / p+1 / p^{\prime}=1$ and $1<p<2$; the integer $k$ involved in this case will be denoted by $k_{m, p}$.

This allows us to state

Proposition 1. Let $k_{m, p}$ be defined for $m \geqq 1$ and $1 \leqq p<2$ as in equations (2.1) below. Then $\mathcal{F}(f * \phi)=\mathcal{F}(f) \cdot \mathcal{F}(\phi)$ for any $\phi \in L^{p^{\prime}}\left(R^{m}\right)$, provided $f \in L^{p}\left(R^{m}\right)$ is such that $F=\mathcal{F}(f)$ is a function of class $\left(\varepsilon^{k_{m, p}}\right)$.

Let us next consider the case $2 \leqq p<\infty$, so that $1<p^{\prime} \leqq 2$. Here $\Phi=\mathfrak{F}(\phi)$ is a function $\Phi \in L^{p}\left(R^{m}\right)$. As before, $\left(r_{n} f\right) * \phi \rightarrow f * \phi$ in $\left(\mathcal{S}^{\prime}\right)$. Further, $\left(F * R_{n}\right) \cdot \Phi \rightarrow F \cdot \Phi$ in $\left(\Phi^{\prime}\right)$ provided merely that $F$ is a function belonging locally to $L^{p^{\prime}}$. So we have

PRoposition 2. If $2 \leqq p<\infty, \mathcal{F}(f * \phi)=\mathscr{f}(f) \cdot \mathcal{F}(\phi)$ for any $\phi \in L^{p^{\prime}}\left(R^{m}\right)$

${ }^{1} D^{q}=\partial a_{1}+\cdots+q_{m} / \partial y_{1}^{a_{1}} \cdots \partial y_{m}^{a_{m}}$ with rank $q_{1}+\cdots+q_{m}$. 
provided $f \in L^{p}\left(R^{m}\right)$ is such that $F=\mathcal{F}(f)$ is a function belonging locally to $L^{p^{\prime}}\left(R^{m}\right)$.

This of course implies, as it should, that the formula holds always in the case $p=2=p^{\prime}$.

The case $p=\infty$ and $p^{\prime}=1$ could also be discussed, but its applications to translational problems are of little interest.

As a final example, let $f \in C_{0}\left(R^{m}\right)$, the Banach space of continuous functions on $R^{m}$ which tend to zero at infinity, the topology being that of uniform convergence. Take any $\mu \in \mathcal{X}^{1}\left(R^{m}\right)$, the space of bounded Radon measures on $R^{m}$, considered as the dual of $C_{0}\left(R^{m}\right)$. In this case, $M=\mathcal{F}(\mu)$ is a function in $(\mathcal{E})$, so that the exchange formula will hold provided $F=\mathcal{F}(f)$ is a measure. For then $r_{n} f \rightarrow f$ uniformly, hence $\left(r_{n} f\right) * \mu \rightarrow f * \mu$ boundedly, and a fortiori in $\left(S^{\prime}\right)$. Further, $\left(F * R_{n}\right) \cdot M \rightarrow F \cdot M$ in $\left(\Phi^{\prime}\right)$, as the following argument shows. If $\alpha(y) \in(\Phi)$,

$$
\begin{aligned}
\left(F * R_{n}\right) \cdot M(\alpha) & =\int_{R^{m}} \alpha(y) M(y) \cdot F * R_{n}(y) d y \\
& =\int_{R^{m}} \alpha(y) M(y) d y=\int_{R^{m}} R_{n}(y-z) d F(z) \\
& =\int_{R^{m}} d F(z) \int_{R^{m}} R_{n}(y-z) \alpha(y) M(y) d y
\end{aligned}
$$

here the inner integral has a fixed bounded support and converges to $\alpha(z) M(z)$ uniformly, so that the whole expression converges to $\int_{R^{m}} \alpha(z) M(z) d F(z)=F \cdot M(\alpha)$. This leads to

Proposition 3. $\mathcal{F}(f * \mu)=\mathcal{F}(f) \cdot \mathcal{F}(\mu)$ for any $\mu \in \mathcal{X}^{1}\left(R^{m}\right)$, provided $f \in C_{0}\left(R^{m}\right)$ is such that $F=\mathcal{F}(f)$ is a measure.

It ois easy to check that the following estimates of the integers $k_{m, p}$ are valid:

$$
k_{1, p}=1 \quad \text { for } 1 \leqq p<2 \text {, }
$$

$$
k_{m, 1}=\text { the smallest even integer satisfying }
$$

$$
2 k_{m, 1}>m,
$$

$k_{m, p}=$ the smallest even integer satisfying

$$
p k_{m, p}>m \quad(1<p<2)
$$

if $m>1$. 
3. Applications to spans of translates. We begin with the case $p=1$.

TheOREM 1. Let $k_{m}=k_{m, 1}$ be defined as in (2.1), let $f \in L^{1}\left(R^{m}\right)$ be given, and let $E_{f}$ be the set of zeros of $F=\mathcal{F}(f) \in(\varepsilon)$. Then the span of translates of $f$ in $L^{1}\left(R^{m}\right)$ contains every function $g \in L^{1}\left(R^{m}\right)$ whose transform $G=\mathcal{F}(g)$ satisfies the conditions:

(i) $G$ is a function in $\left(\varepsilon^{k_{m}}\right)$, and

(ii) $G=0$ on $E_{f}$, and $D^{a} G=0$ on the derived set $E_{f}^{\prime}$ for each symbol of derivation

$$
D^{q}=\partial^{q_{1}+\cdots+q_{m}} / \partial y_{1}^{q_{1}} \cdots \partial y_{m}^{q_{m}}
$$

of rank $q_{1}+\cdots+q_{m} \leqq k_{m}$. When $m=1, k_{m}=1$, and (ii) may be replaced by the assumption

(ii) $)_{1} G$ on $E_{\text {s. }}$.

Remarks. For $m=1$, Beurling [2] had already proved a stronger result as early as 1938 . Related results have been proved more recently by Mandelbrojt and Agmon [3]. Extensions to arbitrary locally compact abelian groups have been made by Helson [4] and Reiter [5]. The proof below has been constructed independently by Schwartz, who has also communicated to the author an alternative demonstration avoiding the use of distributions.

Proof. We recall the following points concerning $L^{\infty}\left(R^{m}\right)$, which in the sequel will always be taken with its weak topology as the dual of $L^{1}\left(R^{m}\right)$, the fundamental bilinear form defining the duality being

$$
\langle f, \phi\rangle=\int_{R^{m}} f(x) \phi^{\smile}(x) d x \quad\left(\phi^{\smile}(x)=\phi(-x)\right) .
$$

Given $\phi \in L^{\infty}\left(R^{m}\right)$, the spectrum $\Lambda_{\phi}$ of $\phi$ is the set of $y \in R^{m}$ such that

$$
\exp 2 \pi i(x, y) \equiv \exp 2 \pi i\left(x_{1} y_{1}+\cdots+x_{m} y_{m}\right)
$$

is the (weak) limit of finite linear combinations of translates of $\phi$. $\Lambda_{\phi}$ is a closed set which is known to be identical with the support of $\Phi=\mathcal{F}(\phi)$ (see Schwartz [6, Théorème IX]). Further, at each isolated point of $\Lambda_{\phi}, \Phi$ is locally a multiple of the Dirac measure at that point.

By the Hahn-Banach theorem, our assertion will be proved if we show that $g * \phi=0$ whenever $\phi \in L^{\infty}\left(R^{m}\right)$ satisfies $f * \phi=0$. Now $f * \phi$ $=0$ implies that $\Lambda_{\phi} \subset E_{f}$. And, by Proposition 1, we are reduced to showing that our hypotheses on $G$ imply that $G \cdot \Phi=0$. This follows by a theorem of Whitney [7] on ideals of differentiable functions by 
virtue of our remarks concerning the nature of $\Phi$ at its isolated points of support.

Finally, for the case $m=1$, (ii) follows already from (ii) by use of Rolle's theorem.

We turn next to the case $1<p<2$. An analogous argument, based upon Proposition 1, yields

Theorem 2. Let $1<p<2$, and let $f \in L^{p}\left(R^{m}\right)$ be such that $F=\mathcal{F}(f)$ is a function in $\left(\varepsilon^{k_{m}, p}\right)$ which vanishes on $E_{f}$. Then the span of translates of $f$ in $L^{p}\left(R^{m}\right)$ contains every $g \in L^{p}\left(R^{m}\right)$ whose transform $G=\mathcal{F}(g)$ satisfies the conditions:

(i) $G$ is a function in $\left(\varepsilon^{k_{m, p}}\right)$,

(ii) $D^{q} G=0$ on $E_{f}^{\prime}, D^{a}$ being as in Theorem $1\left(k_{m, 1}\right.$ being replaced by $k_{m, p}$, of course, and $q=0$ being allowed).

When $m=1$, (ii) can be replaced by

(ii) $1=0$ on $E_{f}^{\prime}$.

Proof. Take any $\phi \in L^{p^{\prime}}\left(R^{m}\right)$, the space dual to $L^{p}\left(R^{m}\right)$, which is orthogonal to all translates of $f$. Then, by Proposition 1, one has $F \cdot \Phi=0$, so that the support $K$ of $\Phi$ satisfies $K \subset E_{f}$. We have to prove (Proposition 1 again) that $G \cdot \Phi=0$. Since $\phi \in L^{p^{\prime}}\left(R^{m}\right)$ and $p^{\prime}<\infty$, it is easy to see that $K$ has no isolated points, so that $K \subset E_{f}^{\prime}$. Whitney's theorem again serves to justify the assertion that $G \cdot \Phi=0$, so that the proof is complete.

Corollary. If $f \in L^{p}\left(R^{m}\right)(1<p<2)$ is such that $F=\mathcal{F}(f)$ is a function in $\left(\varepsilon^{k_{m}, p}\right)$ with a set of zeros having no limiting point, then the translates of $f$ span all $L^{p}\left(R^{m}\right)$.

REMARK. This corollary, together with that following the next theorem, should be compared with Theorem 3.5 of Segal [8] and with the counterexamples due to the same writer (Theorem 3.4 of [8], and [9]).

Turning to the remaining (finite) values of $p$, we can exclude the trivial case $p=2$, passing at once to

THEOREM 3. Let $2<p<\infty$, and let $f \in L^{p}\left(R^{m}\right)$ be such that $F=\mathcal{F}(f)$ is a function belonging locally to $L^{p^{\prime}}\left(R^{m}\right)$. Let $E_{f}$, defined modulo null sets, be the set of zeros of $F$. Then the span of translates of $f$ in $L^{p}\left(R^{m}\right)$ contains every function $g \in L^{p}\left(R^{m}\right)$ whose transform $G=\mathcal{F}(g)$ is a function belonging locally to $L^{p^{\prime}}\left(R^{m}\right)$ which vanishes p.p. on $E_{f}$.

The proof of this follows exactly the same lines as before, appeal being made to Proposition 2 rather than Proposition 1.

CoRollary. If $f \in L^{p}\left(R^{m}\right)(2<p<\infty)$ satisfies the conditions of 
Theorem 3, and if in addition $E_{f}$ is null, then the translates of $f$ span all $L^{p}\left(R^{m}\right)$.

REMARK. This corollary compares naturally with assertion (A) of Segal [9] (the corollary is of course trivially true when $p=2$ ). On the face of things, Segal's hypothesis on $f$ that its transform shall exist means that $f$ is the inverse transform of some function $F \in L^{p^{\prime}}\left(R^{m}\right)$; we require merely that the same is true in the sense of distributions for some $F$ belonging locally to $L^{p}\left(R^{m}\right)$.

We may just observe that the analogue, for $p=\infty$, of Propositions 1 and 2 yields this assertion: Let $f \in L^{\infty}\left(R^{m}\right)$ be such that $F=\mathcal{F}(f)$ is a measure, and let $E_{f}$ be the complement of the support of $F$. Then the span of translates of $f$ in $L^{\infty}$-weak contains veery $g \in L^{\infty}\left(R^{m}\right)$ whose transform $G=\mathcal{F}(\mathrm{g})$ is a measure with support $\subset \subset E_{f}$. In particular, if $E_{f}$ is empty, the weak span of translates of $f$ is all $L^{\infty}\left(R^{m}\right)$.

Finally, by use of Proposition 3, there follows

Theorem 4. Let $f \in C_{0}\left(R^{m}\right)$ be such that $F=\mathcal{F}(f)$ is a measure, and let $E_{f}$ be the complement of the support of $F$. Then the span of translates of $f$ in $C_{0}\left(R^{m}\right)$ contains every $g \in C_{0}\left(R^{m}\right)$ whose transform $G=\mathcal{F}(g)$ is a measure with support contained in $C E_{f}$.

Proof. If $\mu \in \mathcal{X}^{1}\left(R^{m}\right)$ is orthogonal to all translates of $f$, one has $f * \mu=0$. Hence, by Proposition $3, M \cdot F=0, M(y)$, the transform of $\mu$, being a continuous function. Hence $M(y)=0$ on $\mathcal{C} E_{f}$. But then $M \cdot G$ $=0$, that is (Proposition 3 again) $\mu * g=0$, whenever the support of $G$ is contained in $C E_{f}$, whence the assertion, by virtue of the HahnBanach theorem.

Corollary. If $f$ is as in Theorem 3, and if in addition $E_{f}$ is void, $t^{\text {hen }}$ the translates of $f$ span all $C_{0}\left(R^{m}\right)$.

REMARK. The corollary includes as a very special case the result of Agnew [10, Theorem 6.1] concerning the uniform span of translates of a "peak function" $f$ of the form

$$
f(x)= \begin{cases}a(b-|x|) & \text { for }|x| \leqq b, \\ 0 & \text { elsewhere, }\end{cases}
$$

the dimension $m$ being unity. Here $F=\mathcal{F}(f)$ is a continuous function with a discrete set of zeros, so that $E_{f}$ is void. On the contrary, Agnew's result [11, Theorem 7.3], on the uniform span of translates of a simple step function $f$ of the form

$$
f(x)= \begin{cases}1, & -b \leqq x<b, \\ 0, & \text { elsewhere }\end{cases}
$$


does not follow directly from the above corollary because $f$ is not continuous. However, the transform of such an $f$ is again a function $F$ with a discrete set of zeros, so that the corollaries to Theorems 2 and 3 imply that $f$ has its translates spanning $L^{p}\left(R^{1}\right)$ for every $p$ satisfying $1<p<\infty$. These remarks apply also to the $L^{p}$-spans of peak functions, so that we recover as very special cases all the results of Agnew on $L^{p}$-spans in one dimension.

\section{REFERENCES}

1. L. Schwartz, Theorie des distributions, vols. 1 and 2, Paris, Hermann, 1950 and 1951.

2. A. Beurling, Comptes Rendus 8th Congress Scand. Mathematiciens, 1938.

3. S. Mandelbrojt and S. Agmon, Une generalisation du theoreme tauberien de Wiener, Acta Sci. Math. Szeged vol. 12 (1950) pp. 167-176.

4. H. Helson, Spectral synthesis of bounded functions, Arkiv. för Mat. vol. 1 (1951) pp. 497-502.

5. $\mathrm{H}$. Reiter, Investigations in harmonic analysis, Trans. Amer. Math. Soc. vol. 73 (1952) pp. 401-427.

6. L. Schwartz, Analyse et synthese harmoniques dans les espaces de distributions, Canadian Journal of Mathematics vol. 3 (1951) pp. 503-512.

7. H. Whitney, On ideals of differentiable functions, Amer. J. Math. vol. 70 (1948) pp. 635-658.

8. I. E. Segal, The group algebra of a locally compact group, Trans. Amer. Math. Soc. vol. 61 (1947) pp. 69-105.

9. - The span of translates of a function in a Lebesgue space, Proc. Nat. Acad. Sci. U.S.A. vol. 30 (1944) pp. 165-169.

10. R. P. Agnew, Spans in Lebesgue and uniform spaces of translations of peak functions, Amer. J. Math. vol. 67 (1945) pp. 431-436.

11. - Spans in Lebesgue and uniform spaces of translations of step functions, Bull. Amer. Math. Soc. vol. 51 (1945) pp. 229-233.

Birkbeck College, University of London 